\title{
Radiological diagnosis of cerebral venous thrombosis in paediatric age group by Magnetic resonance venography: Pictorial essay
}

\author{
Sarkar P.S. ${ }^{1}$, Bhosale P.R. ${ }^{2}$, Rekha B.P. ${ }^{3}$, Hajari $D^{4}$, Hoisala R.V. ${ }^{5}$ \\ ${ }^{1}$ Dr Partha Sarathi Sarkar, Senior Resident, Department of Radiodiagnosis, St Johns Medical College, Koramangala, \\ Bangalore, ${ }^{2}$ Dr Pravin Ranganath Bhosale, Department of Radiodiagnosis, Assistant Professor, Government Medical \\ College, Miraj, ${ }^{3}$ Dr Rekha B. P (Currently pursuing), St Johns Medical College, Koramangala, Bangalore, ${ }^{4}$ Dr Debadatta \\ Hajari, Senior Resident, Department of Emergency Medicine, St Johns Medical College, Koramangala, Bangalore, \\ ${ }^{5}$ Dr Ravi V Hoisala, Professor, Department of Radiodiagnosis, St Johns Medical College, Koramangala, Bangalore.
}

Address for Correspondence: Partha Sarathi Sarkar, Senior Resident in Radiology, St. John's Medical College, Bangalore. Email: drparthasarathisarkar@gmail.com

\begin{abstract}
Cerebral venous thrombosis (CVT) in paediatric age group is often associated with nonspecific clinical complaints. Underdiagnosis or misdiagnosis of cerebral venous thrombosis can lead to severe consequences. Associated findings and complications include haemorrhagic infarction, cortical laminar necrosis, hypoxic ischemic encephalopathy, parenchymal atrophy and extra-axial haemorrhage. Morbidity and mortality can be significant and long-term neurological sequelae include developmental delay, seizures, sensorimotor and visual deficits. This review highlights the need for early detection of CVT, its associations and complications by Magnetic resonance imaging with Magnetic resonance venography. The various etiologies have also been Discussed.
\end{abstract}

Keywords: CVT, MRI, MR Venography, Paediatrics.

\section{Introduction}

Cerebral venous thrombosis (CVT) in children is a multifactorial disease that, in the majority of cases, results from a combination of prothrombotic risk factors and / or the underlying clinical condition [1]. It is a serious disease that is being increasingly diagnosed, mainly because of more sensitive diagnostic procedures and increasing clinical awareness of the disease. The clinical presentation shows a wide spectrum of symptoms, eg, seizures, papilloedema, headache, lack of consciousness or lethargy, and focal neurological deficits [2]. We present a total of 13 paediatric patients of age group 1-14 years with cerebral venous thrombosis. The study was conducted in St John's Medical College, Bangalore from June 2012 to November 2015

\section{Materials and Methods}

MRI brain and MRV was done on 1.5 Tesla GE HDx machine on all patients. The routine sequences were

Manuscript received: $24^{\text {th }}$ May 2016

Reviewed: $4^{\text {th }}$ June 2016

Author Corrected; $14^{\text {th }}$ June 2016

Accepted for Publication: $29^{\text {th }}$ June 2016
Axial T2W and FLAIR, Sagittal T1W, Axial DWI, Axial GRE and Time of Flight MR venogram sequences. The study was conducted in St John's Medical College, Bangalore from June 2012 to November 2015 on a total of 13 paediatric patients of age group 1 - 14 years with cerebral venous thrombosis were studied. Out of 13 patients, 6 were female and 7 were male (Figure 1).

Out of 13 patients, the most common presentation was headache- 6 , followed by seizure- 4 , diarrhea, vomiting - 3 and altered sensorium - 2 (Figure 2).

The most common sinus involved was transverse sinus in 12 out of $13(92 \%)$, followed by sigmoid sinus in 10 (76\%), superior sagittal sinus in $5(38 \%)$ and straight sinus in $4(30 \%)$, internal jugular vein in $4(30 \%)$, cortical veins in $2(15 \%)$, inferior sagittal sinus in 1 (7\%) and vein of Galen in 1 (7\%) (Figure 3).

The various manifestations seen were venous infarcts in 2 patients out of 13 , cortical laminar necrosis in 1 , 
hypoxic ischemic encephalopathy in 1, subarachnoid hemorrhage in 1, parenchymal haemorrhage in 1, and cerebral atrophy in 1 (Figure 4).

The etiologies were dehydration in 4 cases, nephrotic syndrome in 3 , perinatal asphyxia in 2 , haematological in 2, lupus nephritis in 1 and homocysteinemia in 1 (Figure 5).

\section{Discussion}

CVT in children is a result of complex multifactorial elements which result in a prothrombotic state, usually arising from the interaction of acute and chronic pathologies [13]. The majority of children (65\%) have at least two risk factors with $40 \%$ having more than three risk factors. $[3,18]$ (Table 1).

An Indian study conducted in Nizam's institute Hyderabad showed pediatric population constituted $9.4 \%$ of the study population [15]. Another Indian study conducted between 2001 and 2010 reported that 39 of its 624 CVT patients were children [14].

Cerebral venous system can be divided into two basic components. [4-6]

A) Superficial System;The superficial system comprises of sagittal sinuses and cortical veins and these drain superficial surfaces of both cerebral hemispheres. B) Deep System; The deep system comprises of lateral sinus, straight sinus and sigmoid sinus along with draining deeper cortical veins. Both these systems mostly drain themselves into internal jugular veins.

Superficial cerebral venous system The superficial cerebral veins can be divided into three collecting systems [6]. First, a mediodorsal group draining into superior sagittal sinus (SSS) and the straight sinus (SS); Second, a lateroventral group draining into the lateral sinus; Third, an anterior group draining into the cavernous sinus.These veins are linked by the great anastomotic vein of Trolard, which connects the SSS to the middle cerebral veins. These are themselves connected to the lateral sinus (LS) by the vein of Labbe.

The veins of the posterior fossa may again be divided into three groups: a) Superior group draining into the Galenicsystemb) Anterior group draining into Petrosalsinus c) Posterior group draining into the torcularHerophili and neighbouring transverse sinuses. The veins of the posterior fossa are variable in course and angiographic diagnosis of their occlusion is extremely difficult.

The Superior Sagittal Sinus (SSS) starts at the foramen caecum and runs backwards towards the internal occipital protuberance, where it joins with the straight sinus and lateral sinus to form the torcular Herophili. Its anterior part is narrow or sometimes absent, replaced by two superior cerebral veins that join behind the coronal suture. This fact should be borne in mind while evaluating for cerebral venous thrombosis (CVT). The SSS drain major part of the cerebral hemispheres.

The cavernous sinuses drain blood from the orbits, the inferior parts of the frontal and parietal lobe and from the superior and inferior petrosal sinuses. Blood from them flow into the internal jugular veins.

The straight sinus is formed by the union of inferior sagittal sinus and the great vein of Galen. The inferior sagittal sinus runs in the free edge of falxcerebri and unites with the vein of Galen to form the straight sinus. It runs backwards in the center of the tentorium cerebelli at the attachment of the falxcerebri, emptying into the torcularherophili at the internal occipital protuberance.

The lateral sinuses extend from torcularherophili to jugular bulbs and consist of a transverse and sigmoid portion. They receive blood from the cerebellum, the brain stem and posterior parts of the hemisphere. They are also joined by some diploic veins and small veins from the middle ear. There are numerous LS anatomic variations that may be misinterpreted as sinus occlusion[9].

B) Deep cerebral venous system The deep cerebral veins are more important than superficial veins fromthe angiographic point of view [8]. Three veins unite just behind the interventricular foramen of Monro to form the internal cerebral vein. These include choroid vein, septal vein and thalamostriate vein.

The Choroid vein runs from the choroid plexus of the lateral ventricle. The septal vein runs from the region of the septum pellucidum in the anterior horn of the lateral ventricle and the thalamostriate vein runs anteriorly in the floor of the lateral ventricle in the thalamostriate groove between the thalamus and lentiform nucleus. The point of union of these veins is called the venous angle. The internal cerebral veins of each side run posteriorly in the roof of the third ventricle and unite beneath the splenium of the corpus callosum to form the 
great cerebral vein. The internal cerebral veins, which lie within $2 \mathrm{~mm}$ of the midline, are the most important deep veins since they can be used to diagnose midline shifts. [10]

The great cerebral vein of Galen is a short $(1-2 \mathrm{~cm}$ long), thick vein that passes posterosuperiorly behind the splenium of corpus callosum in the quadrigeminal cistern. It receives the basal veinsand the posterior fossa veins and drains to the anterior end of the straight sinus where this unites with the inferior sagittal sinus.

The basal vein of Rosenthal begins at the anterior perforated substance by the union of anterior cerebral vein, middle cerebral vein and the striate vein [11]. The basal vein on each side passes around the midbrain to join the great cerebral vein.

In summary, blood from the deep white matter of the cerebral hemisphere and from the basal ganglia, is drained by internal cerebral veins. [10] and basal veins of Rosenthal, which join to form the great vein of Galen that drains into the straight sinus. With the exception of wide variations of basal vein, the deep system is rather constant compared to the superficial venous system [12]. Hence their thrombosis is easy to recognize.

Two major pathophysiological mechanisms contribute to the clinical presentation of cerebral venous thrombosis (Figure 6). First, thrombosis of cerebral veins or sinuses can result in increased venular and capillary pressure. As local venous pressure continues to rise, decreased cerebral perfusion results in ischemic injury and cytotoxic edema, disruption of the bloodbrain barrier leads to vasogenicedema, and venous and capillary rupture culminates in parenchymal hemorrhage. (Figure 7)

Thrombosis of cerebral sinuses increases venous pressure, impairs cerebrospinal fluid absorption, and ultimately leads to increased intracranial pressure [16].

Clinical manifestations of CSVT are non-specific and may be subtle. Most of the clinical scenarios occur at all ages and the clinician should consider this diagnosis in a wide range of acute neurological presentations in childhood, including seizures, coma, stroke, headache and raised intracranial pressure.(Figure 8). CSVT may also be an important determinant of outcome in nontraumatic coma $[17,18]$

\section{Case review with imaging findings}

Case 1- Reported here is a 7 months old female who presented with history of status epilepticus and altered sensorium.

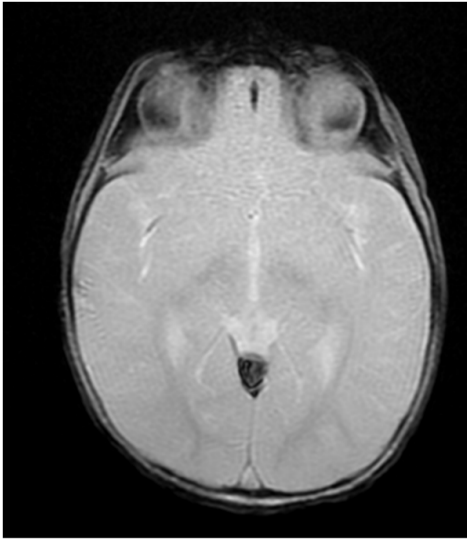

Figure 1a

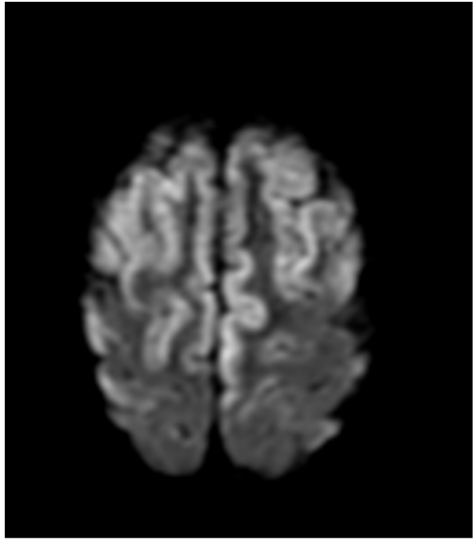

Figure 1b

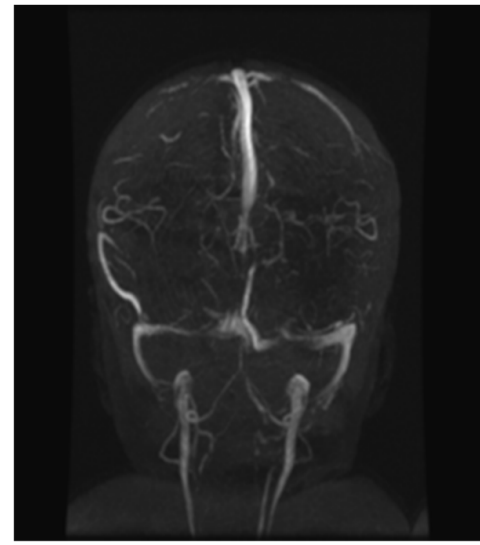

Figure 1c

Axial T2*GRE (Fig 1a), DWI (1b) and MRV (1c) showing partial thrombosis of the vein of Galen with straight sinus extension, thrombosis of the superior sagittal sinus and the draining cortical veins with features of cortical laminar necrosis involving bilateral frontal lobes and post central gyri.

MRI with MRV showed partial thrombosis of the vein of Galen with straight sinus extension,thrombosis of the superior sagittal sinus and the draining cortical veins with features of cortical laminar necrosis involving bilateral frontal lobes and post central gyri. (Figure 1). 
Case 2- Reported here is a 8 yr old female withknown history of ALL who presented with severe headache.

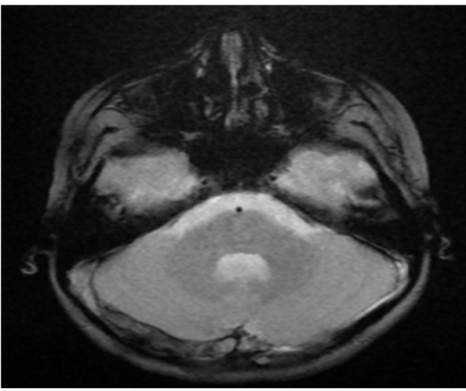

Figure 2a

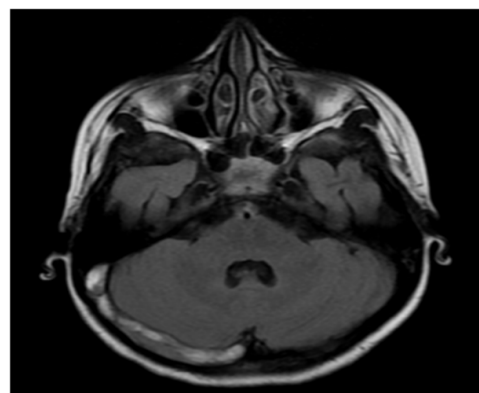

Figure 2b

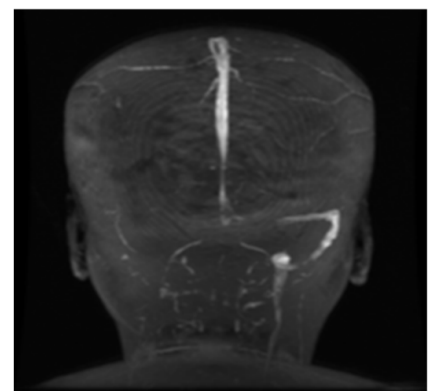

Figure 2c

Axial T2*GRE (Fig 2a) T2 FLAIR (2b) and MRV (2c) showing thrombosis of inferior sagittal sinus, straight sinus, torcularherophili, right transverse sinus, right sigmoid sinus $\&$ jugular bulb.

MRI with MRV showed thrombosis of inferior sagittal sinus, straight sinus, torcularherophili, right transverse sinus, right sigmoid sinus \& jugular bulb (Figure 2).

Case 3- Reported here is a 8 day old male child with history of perinatal asphyxia and focal seizures (right side) on day 2 of birth.

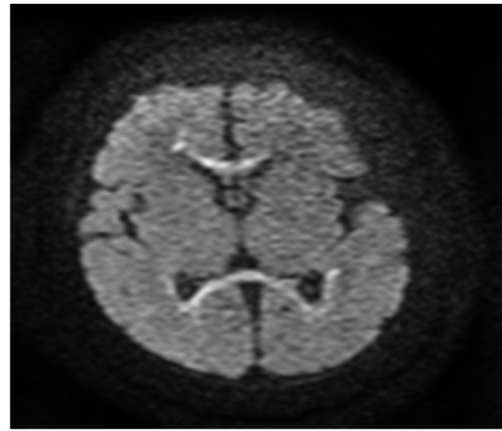

Figure 3a

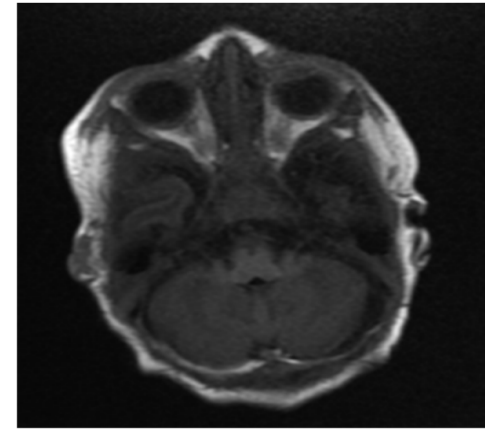

Figure 3b

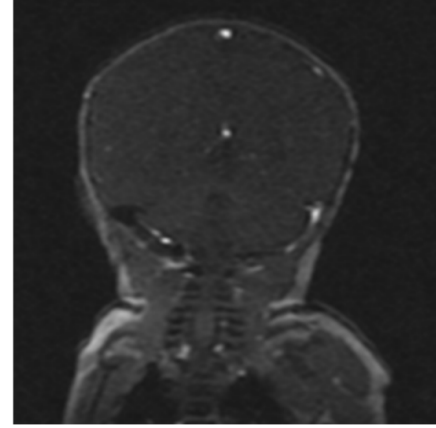

Figure 3c

Axial DWI (Fig 3a), T1 (3b) and MRV (3c) showing restricted diffusion in corpus callosum and right frontal and bitemporal periventricular white matter with bilateral transverse sinus thrombosis.

MRI with MRV showed restricted diffusion in corpus callosum, right frontal and bitemporal periventricular white matter with bilateral transverse sinus thrombosis (Figure 3).

Case 4- Reported here is a 10 day old male child with history of diarrhoea, failure to thrive, severe dehydration and subtle seizures.

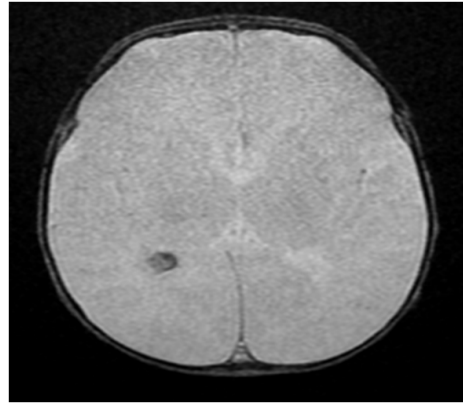

Figure 4a

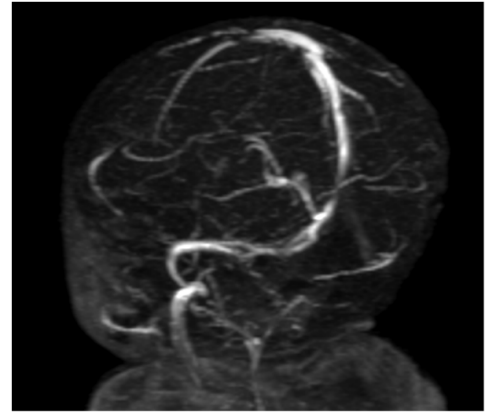

Figure 4b

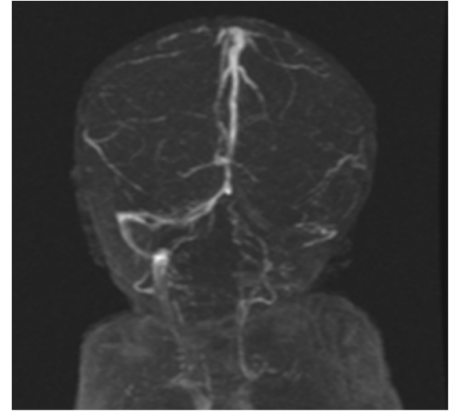

Figure 4c 
Axial GRE (4a) and MRV (4b) showing small focus of restricted diffusion with blooming on GRE in right choroid plexus suggestive of Choroid plexus bleed / cyst with haemorrhage.Filling defect in the left transverse sinus, left sigmoid sinus, proximal right transverse sinus, at the confluence and distal small segment of superior sagittal sinus suggestive of thrombosis

MRI with MRV showed a small focus of restricted diffusion with blooming on GRE in the right choroid plexus suggestive of choroid plexus bleed.Filling defect in the left transverse sinus, left sigmoid sinus, proximal right transverse sinus, at the confluence and distal small segment of superior sagittal sinus suggestive of thrombosis (Figure 4).

Case 5- Reported here is a 7 day old male child with history of lethargy and perinatal asphyxia.

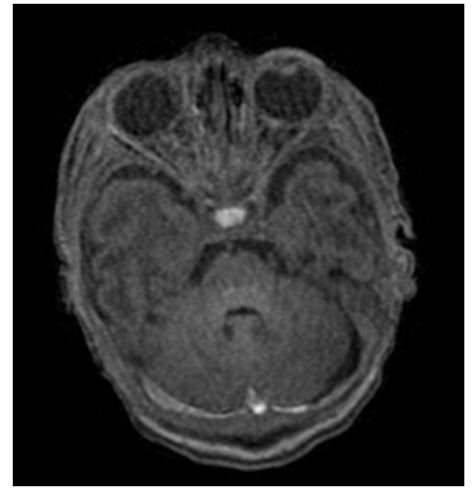

Figure 5a

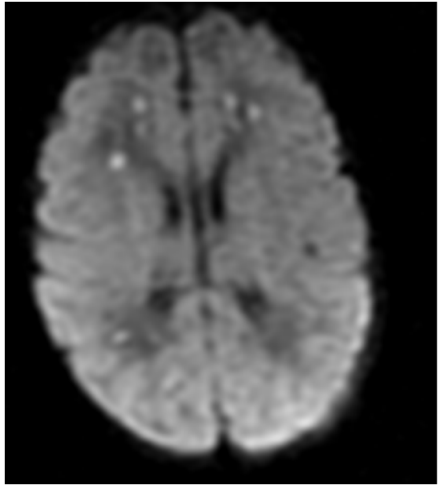

Figure 5b

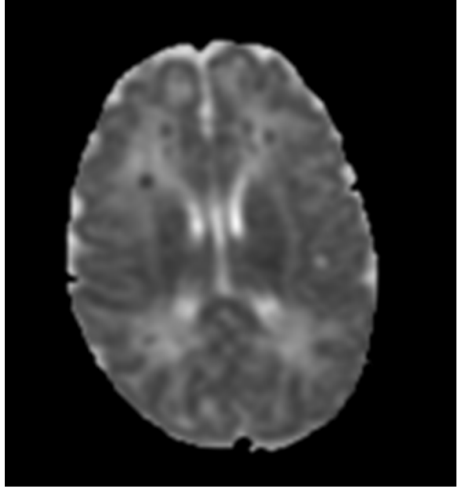

Figure 5c

Axial T2 FLAIR (Fig 5a), DWI (5b) and ADC (5c) showing loss of flow voids in bilateral transverse sinuses with blooming on GRE.- cerebral venous thrombosis.Foci of diffusion restriction in bilateral frontal and right peritrigonal white matter- venous infarcts

MRI with MRV showed loss of flow voids in bilateral transverse sinuses with blooming on GRE suggestive of thrombosis.Foci of diffusion restriction in bilateral frontal and right peritrigonal white matter suggestive of venous infarcts (Figure 5).

Case -6- Reported here is a 8 month old male child who presented with history of vomiting, excessive cry, cough and seizures.

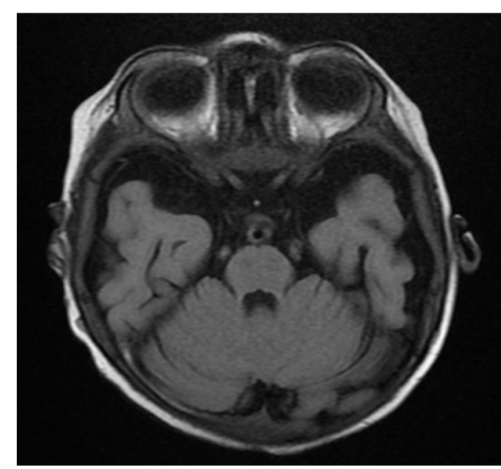

Figure 6a

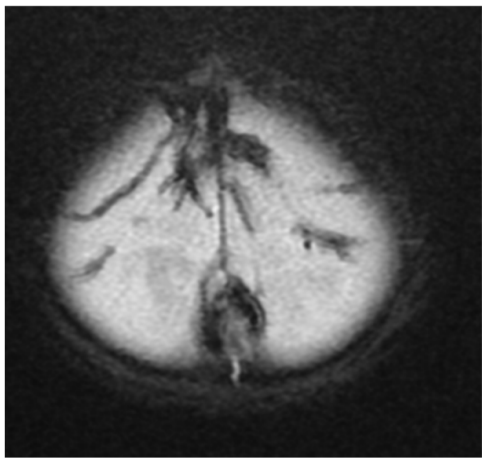

Figure 6b

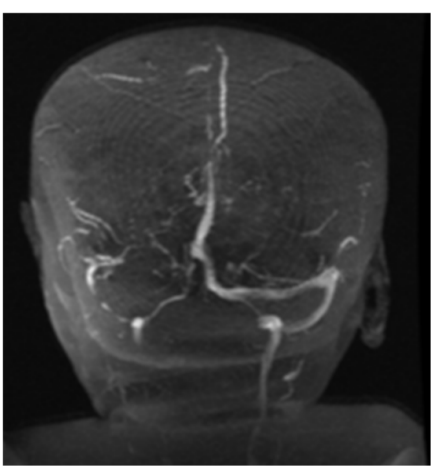

Figure 6c

Axial T2 FLAIR (Fig 6a ), GRE (6b) and MRV (6c) showing significant fronto temporal atrophy with filling defect in the superior sagital sinus, bilateral transverse, right sigmoid sinus and partly the straight sinus with blooming on GRE. 
MRI with MRV showed significant fronto temporal atrophy with filling defect in the superior sagittal sinus, bilateral transverse, right sigmoid sinus and partly the straight sinus with blooming on GRE suggestive of thrombosis (Figure 6).

Case 7- Reported here is a 14 yr old male with history of headache and vomiting.

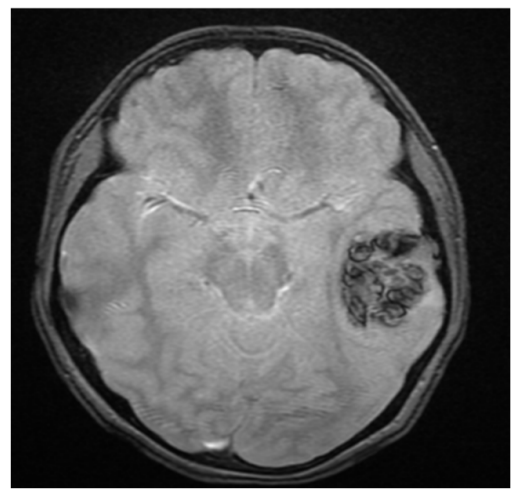

Figure 7a

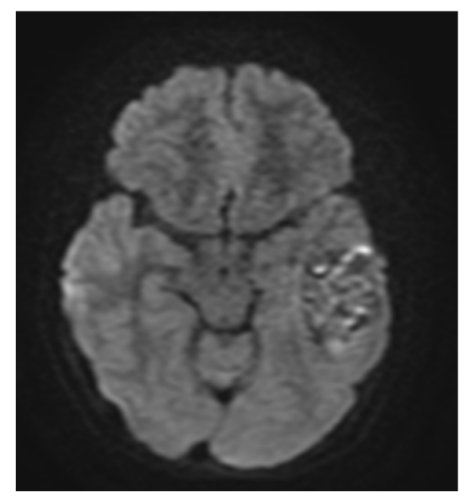

Figure 7b

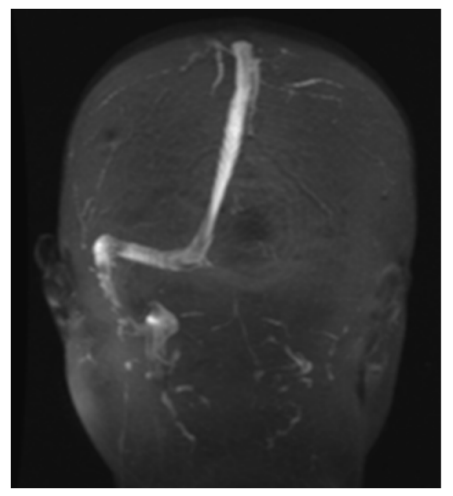

Figure 7c

Axial T2*GRE (7a), DWI (7b) and MRV (7c) showing left transverse and sigmoid sinus thrombosis with hemorrhagic venous infarct in the left temporal lobe.

MRI with MRV showed left transverse and sigmoid sinus thrombosis with hemorrhagic venous infarct in the left temporal lobe (Figure 7).

Case 8- Reported here is a $5 \mathrm{yr}$ old female with history of nephroticsyndrome with worsening of headache, irritability and photophobia.

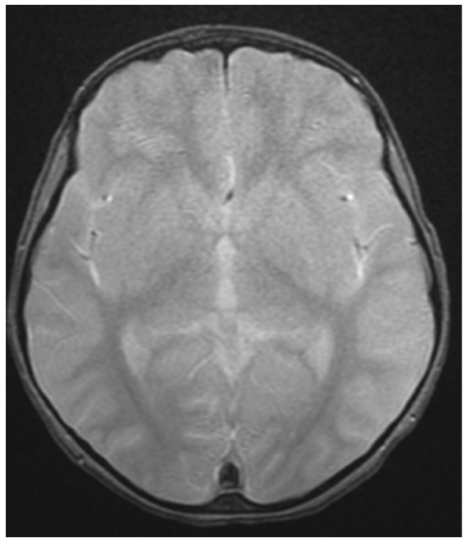

Figure 8a

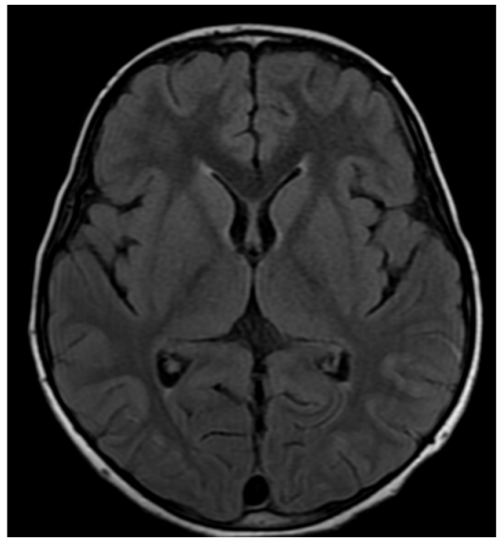

Figure 8b

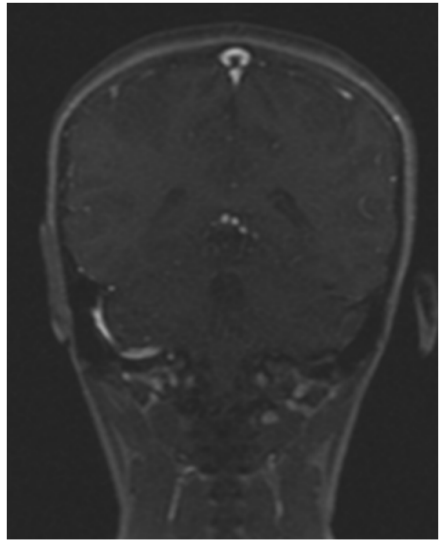

Figure 8c

Axial T2*GRE (8a), T2 FLAIR (8b) and MRV (8c) showing thrombosis of superior sagittal sinus just proximal to the confluence,torculaherophili, left transverse sinuses $\&$ the left sigmoid sinus.

Left posterior temporal cortical vein involvement was also noted.Sulcal T2 FLAIR hyperintensity in left temporoparietal region - suggestive of SAH.

MRI with MRV showed thrombosis of superior sagittal sinus just proximal to the confluence,torcularherophili, left transverse sinuses \& the left sigmoid sinus. Left posterior temporal cortical vein involvement was also noted. Sulcal T2 FLAIR hyperintensity in the left temporoparietalregion suggestive of SAH. (Figure 8). 


\section{Learning Points}

- Cerebral venous thrombosis (CVT) in paediatric age group is often associated with varied clinical presentations and etiologies.

- Findings,associations and complications of cerebral venous thrombosis can be accurately diagnosed by Magnetic resonance imaging with Magnetic resonance venography.

\section{Funding: Nil, Conflict of interest: Nil}

Permission from IRB: Yes

\section{References}

1. Armstead WM, Cines DB, Higazi AAR. Plasminogen Activators Contribute to Impairment of Hypercapnic and Hypotensive Cerebrovasodilation After Cerebral Hypoxia/Ischemia in the Newborn Pig. Stroke 2005;36: 2265-69.

2. Zahuranec DB, Brown DL, Lisabeth LD, Morgenstern LB. Is It Time for a Large, Collaborative Study of Pediatric Stroke?. Stroke 2005;36: 1825-29.

3. Heller C, Heinecke A, Junker R et al. Cerebral venous thrombosis in children: a multifactorial origin. Circulation.2003Sep16;108(11):1362-7.Epub2003Aug 25.

4. Sutton D., Stevens J.: Vascular Imaging in Neuroradiology in Textbook of radiology and Imaging, volume 2 by Churchill Livingstone New York 2003, pp1682-87.

5. Ryan S.P., Mc Nicholas M.M.J., Central Nervous system in Anatomy for diagnostic Imaging by W.B. Saunders Company Ltd. London. 1998, pp 77-80.

6. Kido DK, Baker RA, Rumbaugh Calvin L. Normal Cerebral Vascular Anatomy. In: Abrams Angiography, Vascular and Interventional Radiology by Abrams HL, Third Edition. Little, Brown and Company, Boston. USA. 1983 pp 257-68.

7. Einhaupl KM, MasuhrF.Cerebral Venous and Sinus thrombosis - an update Eur J Neurol 1994; 1: 109 - 26.
8.Meder JF, Chiras J. Roland J, Guinet P, Bracard S, Bargy F. Venous territories of the brain. J Neuroradiol $1994 ; 21: 118-33$.

9. Dora $\mathrm{F}$ and Zileli T: Common Variations of the lateral and occipital sinuses at the confluence sinuum. Neuroradiology 1980; $20: 23$ - 7 .

10. Taveras J.M.: Angiography in Neuroradiology Third Edition. Baltimore: Williams \& Wilkins. 1996 pp 998.

11. Wolf, B.S., Newman, C.M. and Schlesinger, B. The diagnostic value of the deep cerebral veins in cerebral angiography. Am J Radiol. 1962;87:322.

12. Wolf B S, Huang Y P, Newman C.M. The superficial Sylvian venous drainage system.Am J Radiol.1963; 89:398.

13Millichap J. Recognizing Cerebral Venous Sinus Thrombosis. AAP Grand Rounds. 2009;22(6):64-64.

14. Pai N, Ghosh K, Shetty S. Hereditary thrombophili as in cerebral venous thrombosis: A study from India. Blood Coagul Fibrinolysis. 2013;24 (5):540-3.

15.Narayan, Deekshanti, et al. "Risk factors, clinical profile, and long-term outcome of 428 patients of cerebral sinus venous thrombosis: insights from Nizam's Institute Venous Stroke Registry, Hyderabad (India)." Neurology india60.2 (2012): 154.

16. Piazza, Gregory. "Cerebral venous thrombosis." Circulation 125.13 (2012): 1704-1709.

17. S. C, Chuang NA. Pediatric Cerebral Sinovenous Thrombosis: Risk Factors and Complications. Journal Watch Neurology 2001;10:78-80.

18. Alper I. Dai Department of Pediatrics, Shcool of Medicine, Gaziantep Üniversity, Gaziantep, Turkey, November 2006 ;Vol. 56, No. 1.

\section{How to cite this article?}

Sarkar P.S., Bhosale P.R., Rekha B.P., Hajari D, Hoisala R.V. Radiological diagnosis of cerebral venous thrombosis in paediatric age group by Magnetic resonance venography: Pictorial essay. Int J Pediatr Res.2016;3(7):536542.doi:10.17511/ijpr.2016.i07.13. 